\title{
Modelling the rotational modulation of the Sun as a star
}

\author{
A. F. Lanza ${ }^{1}$, M. Rodonò ${ }^{2}$, I. Pagano ${ }^{1}$, P. Barge ${ }^{3}$, and A. Llebaria ${ }^{3}$ \\ 1 INAF, Osservatorio Astrofisico di Catania, Via Santa Sofia, 78, Città Universitaria, 95123, Catania, Italy \\ 2 Dipartimento di Fisica e Astronomia, Università degli Studi, Via Santa Sofia, 78, Città Universitaria, 95123, Catania, Italy \\ 3 LAM, Laboratoire d'Astrophysique de Marseille, BP 8, 13376 Marseille Cedex 12, France \\ e-mail: mrodono@ct.astro.it;ipagano@ct.astro.it; pierre.barge@oamp.fr; antoine.1lebaria@oamp.fr
}

Received 19 November 2002 / Accepted 7 March 2003

\begin{abstract}
We analyse the time variability of the total solar irradiance (TSI) as measured by VIRGO/SoHO in order to model the variability of the Sun as a star. Apart from the phases near the minimum at the beginning of activity cycle 23 , the period of the rotational modulation is significantly different from the solar synodic period as a consequence of the growth and decay of active regions on time scales shorter than a solar rotation. In order to model the variability of the TSI, we have considered the contributions of discrete active regions and a uniformly distributed background emission. To reproduce the rotational modulation of the TSI, we used three active regions, the areas and coordinates of which were changed every seven days to account for their evolution. The simultaneous presence of dark spots and bright faculae was considered by means of appropriate contrast functions which took into account the observed center-to-limb dependence of their contrast with respect to the unperturbed photosphere. The method proved to be capable of modelling the variability of the TSI on time scales going from 7-10 days up to the solar cycle. The relative amplitude of the residuals was of the order of $(1-2) \times 10^{-4}$ with the larger values observed during the phases of maximum solar activity of cycle 23. The application of a similar technique to solar-like stars, such as those that will be observed by the next generation of space-borne photometers, should allow us to minimize the effects of stellar magnetic activity on the detection of planetary transits. Moreover, the availability of long-term highly accurate light curves will allow us to measure stellar rotation period, detect stellar activity cycles, and derive information on the inclination of the stellar rotation axis. The location of the active regions and their irradiance properties can also be retrieved with moderate accuracy from single-band light curves. However, a combination of multi-band photometry and spectroscopy will allow us to constrain some of the free parameters of the model and improve the mapping of stellar surfaces.
\end{abstract}

Key words. Sun: activity - Sun: rotation - stars: activity - stars: rotation - stars: planetary systems

\section{Introduction}

The surface brightness of the Sun and solar-like stars is a complex function of the position on the disk and the time because of the fluctuations associated with convection, p-mode oscillations and magnetic fields. Specifically, solar active regions, arising from photospheric concentration of magnetic flux, contain both bright faculae and dark sunspots. The time evolution of the magnetic field in each active region, the solar rotation and the long-term changes of magnetic activity level associated with the 11-year cycle make the changes of the flux integrated over the solar disk a very complex function of the time. Radiometers on board of several satellites have monitored the changes of the bolometric flux of the Sun since the late seventies (e.g., Chapman 1987; Fröhlich \& Lean 2002). The bolometric flux measurements are usually converted into total solar irradiance (TSI) values, which are independent of distance and other systematic effects, thus revealing the intrinsic variability of the Sun (cf. Fröhlich et al. 1997a). Most of the TSI time

Send offprint requests to: A. F. Lanza, e-mail: nlanza@ct.astro.it variability on time scales of the order of the day or tens of days was explained by models that accounted for the bolometric flux changes produced by the active regions observed on the solar disk by means of empirically derived proxy irradiance functions for sunspots and faculae (e.g., Sofia et al. 1982; Chapman 1987). The modelling of the variability on time scales comparable with the 11-yr activity cycle required the introduction of a uniformly distributed component likely to be associated with the network outside active regions (Foukal \& Lean 1988; Foukal et al. 1991).

The recent TSI data from the VIRGO experiment on board the satellite SoHO are of significantly higher quality than those of previous instruments. Moreover, the sunphotometers of the VIRGO experiment have provided us with simultaneous data in three optical passbands centered at 402, 500 and $803 \mathrm{~nm}$ with a band width of $5 \mathrm{~nm}$, allowing to study the wavelength dependence of flux changes (Fröhlich et al. 1997a). The VIRGO data nicely confirm the validity of the above mentioned modelling approach, i.e., that the active regions are responsible for most of the variability of the TSI, as well as of the fluxes simultaneously monitored by the sunphotometers, on time scales 
ranging from a few days up to a few hundred days (see, e.g., Fligge et al. 1998; Unruh et al. 1999; Fligge et al. 2000).

Stars with an outer convective envelope and a sufficient rotation rate to excite a solar-like dynamo are expected to show the same kind of variability of the Sun (e.g., Godoli 1968; Parker 1979). Ground-based optical measurements are limited to an accuracy of a few $10^{-3}$ mag which is about one order of magnitude larger than the typical amplitude of the solar variability. However, several stars with a color index $0.45 \lesssim$ $B-V \lesssim 1.2$ are observed to be variable both on a year-to-year basis and on a seasonal time scale. The correlation with the chromospheric proxy indicators suggests that a solar-like picture is indeed appropriate and that the optical variability can be explained as the result of active region growth and decay and rotational modulation (Radick et al. 1998).

In the forthcoming years the availability of space-borne photometers dedicated to stellar observations will provide us with thousands of optical time series going from one month up to a few years with a sampling rate from a few seconds to a few tens of minutes and an accuracy of $10^{-6}-10^{-5} \mathrm{mag}$ (Baglin et al. 2001; Favata \& Aigrain 2002). The main purpose of such instruments is to detect stellar oscillations and to find out Earth-like planets orbiting stars through the periodic light dimming produced by their transits across the disk of the parent star (e.g., Jenkins et al. 1996; Defay et al. 2001; Jenkins 2002).

The planned missions dedicated to stellar photometry include COROT, KEPLER and Eddington. They should be launched between 2005 and 2008. For instance, COROT will perform CCD photometry of stellar fields providing also time series of wide-band color index variations. The solar data are bolometric data, however, because the total solar variability closely mimics the changes in the optical band (e.g., Fröhlich et al. 1997a; Fligge et al. 1998), they can be used as a good proxy for future stellar time series. In such a way, we can test possible techniques for modelling the variability of solar-like stars to reduce the impact of magnetic activity on the detection of planetary transits. Moreover, the analysis of time series should provide information on the rotation period and the inclination of the rotation axis of solar-like stars, as well as on their surface differential rotation and activity cycles, when a long-term coverage is available.

The magnetically active stars studied so far are single objects or components of close binaries with flux variation amplitudes of the order of $0.1 \mathrm{mag}$ in the optical passbands. Their light variations are modelled by assuming huge, dark regions on their photospheres (Vogt 1983; Rodonò et al. 1986; Henry et al. 1995). However, when modelling the variability of the Sun, the activity level of which is much lower, it is necessary to account for the presence of faculae and for a diffuse network. Moreover, the area of the solar active regions is always smaller than $1-2 \%$ of the solar surface, so that a simpler approach can be adopted to treat the variability. Its application allows us to model the effects of rotational modulation and active region intrinsic evolution on time scales ranging from about one week up to the solar activity cycle. The method is effective in reducing the amplitude of the solar flux variability, up to an order of magnitude, while leaving unaffected the variability on short scale, due to short-lived active regions with characteristic time scales of about $1-5 \mathrm{~d}$.

In the next section, we present the main characteristics of the VIRGO/SoHO TSI data set and analyse it with an approach we adopted for studying the time series of active stars. In Sect. 3, we introduce our method to model the rotational modulation of the Sun as a star, and in Sect. 4 we present the results of its application. In Sect. 5 we discuss the basic assumption of our method and consider its applicability to other stars. We address its advantages and drawbacks and present a preliminary application to the problem of planetary transit detection. The main conclusions of our study are summarized in Sect. 6.

\section{The total solar irradiance data set and its variability}

The time series that we analyse in the present study is the level 2.0 series of TSI made available by the VIRGO team on the official web site of the mission ${ }^{1}$. The VIRGO experiment is based on two radiometers DIARAD and PMO6-V, each of which has two channels, one in nearly continuous operation and the other used occasionally to evaluate the degradation of the operating channel due to the exposure to the solar radiation (see Fröhlich et al. 1995 and Fröhlich et al. 1997a, for details). Several corrections must be applied to the measurements to derive the intrinsic solar irradiance and, unfortunately, not all of them are known to the desirable level of accuracy.

The irradiance of the Sun as measured by the radiometers was corrected for the changes in the infrared thermal radiation of the environment within each instrument and then normalized to a reference distance of $1 \mathrm{AU}$ and zero orbital velocity in order to reveal the intrinsic variability of the Sun. The effect of the long-term degradation of each radiometer, which was measured using the occasionally exposed channel, was corrected. This correction was performed for both types of radiometers separately, yielding two independent time series which were then combined into a more reliable estimate of the TSI with a reduced long-term uncertainty. Finally, a comparison with the data of other radiometers and the indication coming from empirical models of the TSI changes were applied to reduce further residual systematic long-term trends. This work led to the so-called level 2.0 time series of the TSI, which is the best approximation currently available for the solar intrinsic bolometric irradiance variations (Fröhlich et al. 1997a; Fröhlich 2002).

The time series analysed in the present study consists of one TSI measurement per hour, each measurement having a relative accuracy of $2.0 \times 10^{-5}$. No obvious outliers are present in the level 2.0 data, in contrast to the DIARAD data set analysed by Jenkins (2002). However, several gaps are present, going from occasional gaps not exceeding a few hours, up to the four month gap due to the loss of the satellite tracking in 1998.

A first analysis of the characteristic time scales of variability contained in the TSI time series can be performed by computing the so-called pooled variance of the data

\footnotetext{
1 http://virgo.so.estec.esa.nl/; data are freely available provided the original contribution of the VIRGO team is acknowledged.
} 


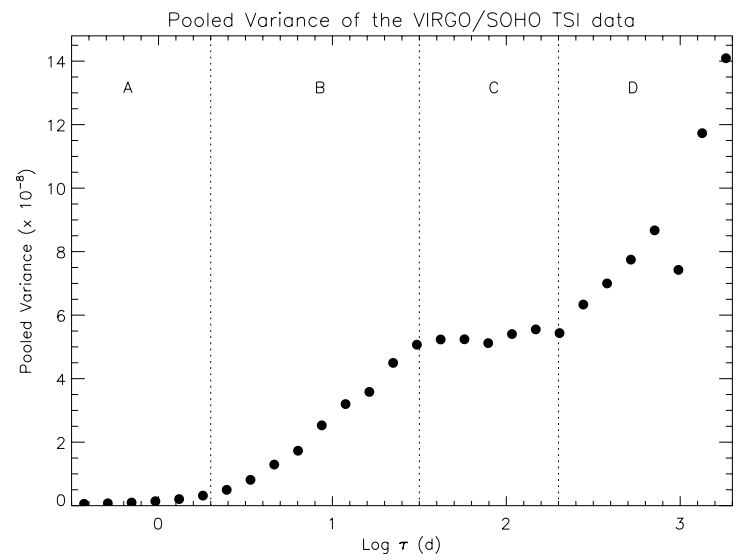

Fig. 1. The pooled variance of the relative variations of the TSI as a function of the time interval $\tau$ chosen to bin the data. The relative variations are computed with respect to a reference value $S_{0}=$ $1367.3 \mathrm{~W} \mathrm{~m}^{-2}$. The main ranges of variation of the pooled variance are indicated with A, B, C and D (see the text).

(Dobson et al. 1990; Donahue \& Baliunas 1992; Donahue 1993). For a given time interval, the data are binned into segments having the length of that interval and the average variance of the set of these segments, that is the pooled variance, is computed. The pooled variance is plotted as a function of the time interval length in Fig. 1, where four ranges of variation can be identified. For the shortest time scales $(\leq 2 \mathrm{~d})$, the pooled variance increases steadly, until a clear slope change appears at $2 \mathrm{~d}$, marking the beginning of another contribution to the variance of the data. We may attribute the increase of the variance for time scales shorter than $2 \mathrm{~d}$ to the presence of the flux fluctuations associated with the supergranular convection which levels off at about $2 \mathrm{~d}$, at approximately the turnover time of its largest convective eddies (cf. Fröhlich et al. 1997b). For longer time scales, first the active region evolution and then the rotational modulation dominate the increase of the variance up to about 30-33 d. The subsequent levelling off of the pooled variance indicates that for time intervals between $\sim 30 \mathrm{~d}$ and $\sim 200 \mathrm{~d}$ there is no other process significantly increasing the variance of the data. For time scales longer than about $200 \mathrm{~d}$, the contribution of the intrinsic evolution of large activity complexes dominates the solar variability (e.g., Donahue \& Keil 1995) and at larger time scales the changes due to the 11-yr activity cycle can be clearly seen. We notice that the number of intervals over which the pooled variance is calculated is quite small in the right part of the range $\mathrm{D}(<8-10)$, which gives rise to sizeable statistical fluctuations of its values with respect to the better sampled ranges A, B and C.

The pooled variance analysis is independent of the shape of the flux time variation. Since we are interested in modelling the rotational modulation, it is useful to apply a different tool to look for a periodicity of the data in the characteristic time range of solar active region rotation. The latitude variation of the activity belts and the different mean lifetime of the surface features, as a function of the activity cycle phase, produce a variation of the period of the rotational modulation along the 11-yr cycle (e.g., Donahue \& Keil 1995). This effect is worth to be taken into account since our time series goes from January 1996 to September 2001, i.e., from the activity minimum, at the beginning of cycle 23 , up to its maximum and beyond. A useful tool to study the time variability of the rotational modulation is provided by the Morlet wavelet, as described in detail by Hempelmann \& Donahue (1997) and Hempelmann (2002). We computed the Morlet wavelet amplitude for the entire TSI time series by adopting the definition of Hempelmann \& Donahue (1997). The relative amplitude of the wavelet transform is plotted in Fig. 2, together with the data sequence, so that a readily straightforward comparison between them is possible. Our wavelet transform amplitude was computed for a rotation period ranging from $22.0 \mathrm{~d}$ to $33.5 \mathrm{~d}$, that is compatible with the time series variance showing a monotonous increase up to $\sim 30-33 \mathrm{~d}$. The time resolution of the wavelet transform is of about $\sim 300 \mathrm{~d}$, as in Hempelmann \& Donahue (1997). This choice represents a good compromise between rotation period and time resolution, which are inversely correlated. From Fig. 1 we deduce that the growth and decay of the activity complexes become significant for time scales longer than about $200-250 \mathrm{~d}$, making it not advisable to degrade the time resolution in order to increase the period resolution because the intrinsic evolution of the surface brightness inhomogeneities would prevent a better period determination. Therefore, in the present analysis the resolution in the rotation period determination is limited to $\sim 0.5 \mathrm{~d}$.

During the phase of solar cycle minimum (1996-1998), the peak amplitude of the wavelet is $\sim 40 \%$ of its maximum, but the period is well defined at about $27.7 \mathrm{~d}$, which corresponds to the synodic rotation period of the Sun in the latitude range of the sunspot groups. The large data gaps in 1998 are likely to be responsible for the period shifts and for the splitting observed from 1998.0 to 1999.0 , so that the wavelet analysis in this time interval can not be trusted. Starting from 1999.1, the data continuity is restored. The results, however, can be regarded with confidence only from mid 1999, because the wavelet feels the effects of the gap up to 150-200 d after it ended. In the time interval from 1999.5 to the beginning of 2001, the wavelet transform amplitude is about two times larger than during the minimum phase, because the amplitude of the rotational modulation due to active regions is now larger. However, the simple structure of the wavelet transform is now lost. There is a splitting of the amplitude into two ridges, one centered around a period of $26.2 \mathrm{~d}$ and another around $31 \mathrm{~d}$, with the latter having the largest amplitude. Such a splitting can be interpreted as a consequence of the phase shifts produced by the growth and decay of activity complexes at different longitudes, and over times scales shorter than the time resolution of the Morlet wavelet, as discussed by Hempelmann (2002). Therefore, when the Sun is near the maximum of the 11-yr cycle, the rotational modulation signal can be masked by the active region evolution. A similar conclusion was reached by LaBonte $(1982,1984)$ by the analysis of the absolute value of the magnetic flux and the $2.8 \mathrm{GHz}$ solar flux time variations, the latter being a good tracer of sunspot groups. The rotation period as derived by means of standard Fourier analysis techniques applied to $1 \mathrm{yr}$ data sets, turned out to be highly variable with values differing from the true solar synodic period up to about 6 d. Donahue \& Keil (1995) pointed out that a 


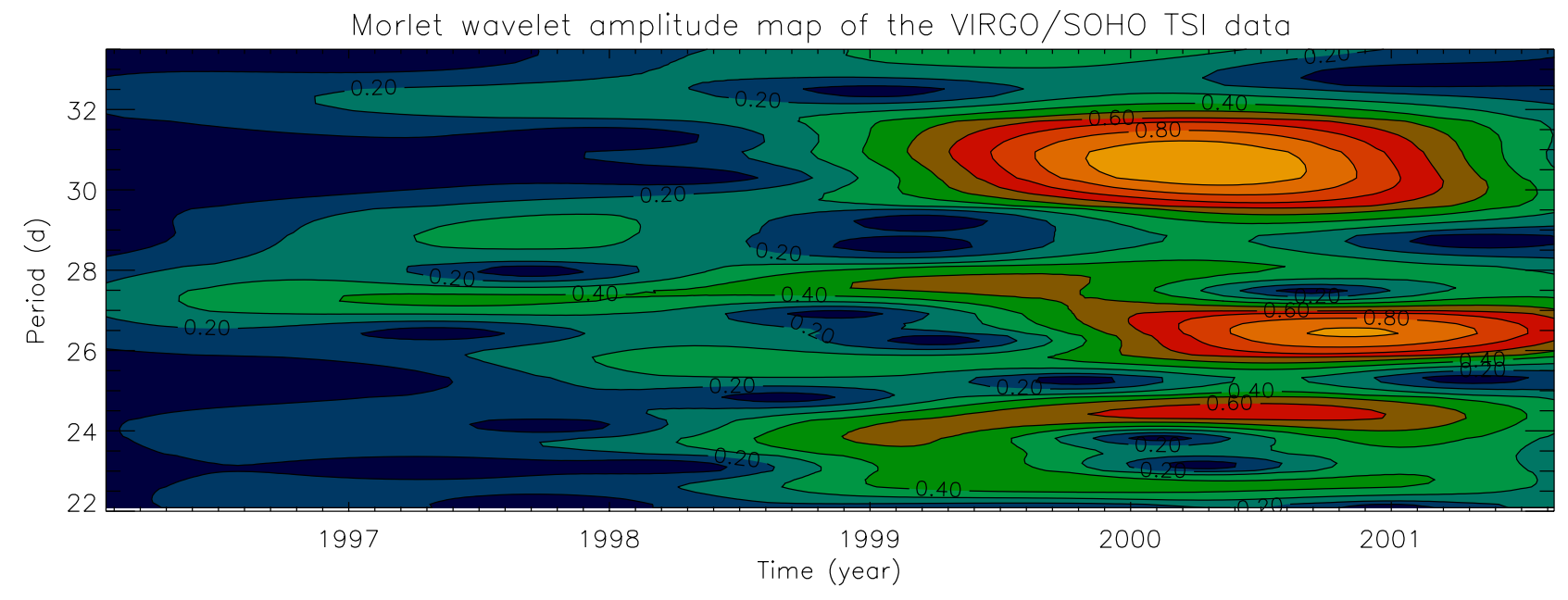

The VIRGO/SOHO Total Solar Irradiance data

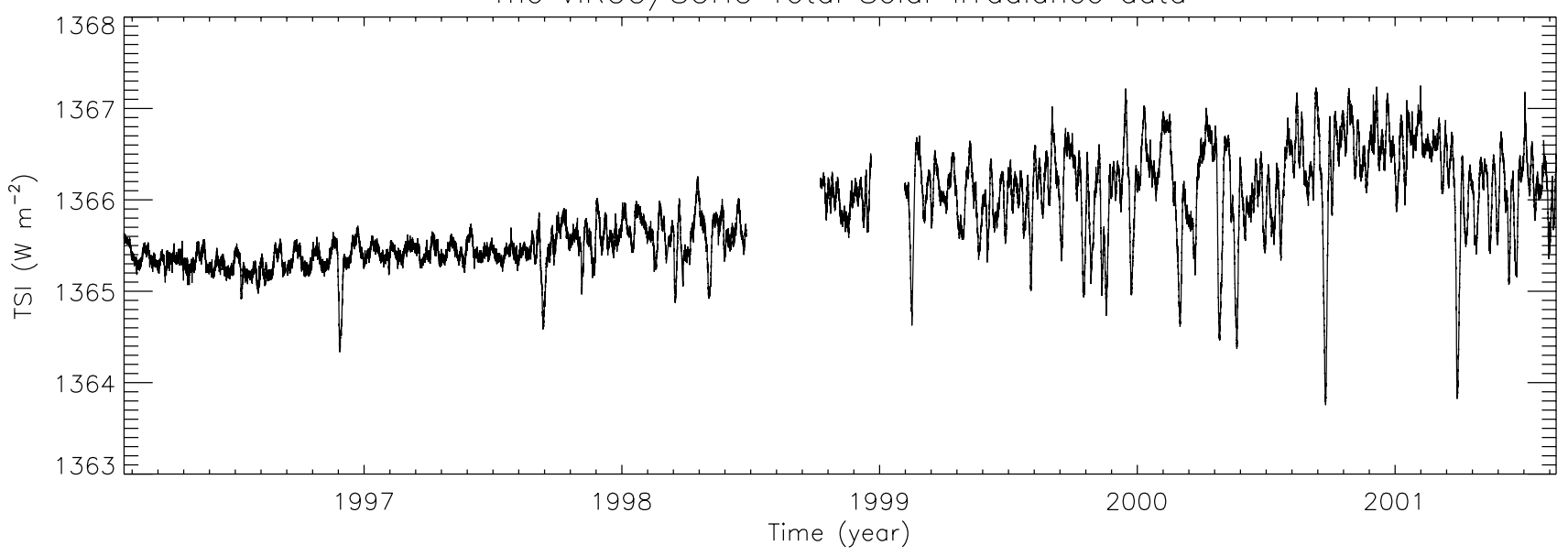

Fig. 2. Upper panel: the amplitude of the Morlet wavelet of the TSI time series normalized to its maximum value. Lower panel: the TSI time series of level 2.0 as derived by the measurements of VIRGO/SoHO described in Sect. 2.

crucial parameter for the proper determination of the rotational modulation is the length of the data set over which the Fourier spectrum is computed and suggested that it should not exceed 150-200 d for the Sun in order to detect systematic changes related to the evolution of the activity cycle. In view of such considerations, we have re-analysed the TSI time series after splitting it into thirteen subsets of length $158 \mathrm{~d}$. In order to attain a better estimate of the rotational modulation period and of the confidence level of the detected periodicity, we applied the Lomb-Scargle periodogram method to each subset (Scargle 1982; Horne \& Baliunas 1986). The results are listed in Table 1, where the MJD of the center of each subset (the first column), the year (the second column) and the period $P_{\text {mod }}$ corresponding to the main peak of the periodogram of the data in the subset, are given. The confidence level of all the peaks is larger than $99.9 \%$. The effective resolution of the period determination is of the order of $1.0 \mathrm{~d}$, except for the sixth and the seventh subsets for which the large data gaps degrade the resolution by a factor of about two. The results presented in Table 1 reinforces the conclusion that the measured rotational modulation period is highly variable and cannot be determined accurately, especially during the phases of high activity, due to the growth and decay of active region complexes, which
Table 1. The period corresponding to the highest periodogram peak in each of the subsets of $158 \mathrm{~d}$ derived by dividing the entire TSI series into 13 time intervals.

\begin{tabular}{ccc}
\hline \hline $\begin{array}{c}\text { MJD } \\
\left(J D-2.4 \times 10^{5}\right)\end{array}$ & Year & $\begin{array}{c}P_{\text {mod }} \\
(\mathrm{d})\end{array}$ \\
\hline 50180.77 & 1996.27 & 26.2 \\
50338.77 & 1996.70 & 26.7 \\
50496.77 & 1997.13 & 27.2 \\
50654.77 & 1997.56 & 31.4 \\
50812.77 & 1998.00 & 30.3 \\
50970.77 & 1998.43 & 29.3 \\
51128.77 & 1998.86 & 25.0 \\
51286.77 & 1999.30 & 24.5 \\
51444.77 & 1999.73 & 28.5 \\
51602.77 & 2000.16 & 29.9 \\
51760.77 & 2000.59 & 31.9 \\
51918.77 & 2001.03 & 22.0 \\
52076.77 & 2001.46 & 26.2 \\
\hline
\end{tabular}

appear randomly in time and longitude on typical time scales of $\approx 200 \mathrm{~d}$. Therefore, any modelling of the rotational 
modulation of the TSI during the 1996-2001 period must assume the rotation period as an additional free parameter, the best value of which should be determined with the method described in the next section.

\section{The model of the solar rotational modulation}

In order to model the variation of the solar irradiance due to active regions, we adopted the approach described by Oster et al. (1982) and Sofia et al. (1982). The difference between the solar irradiance in the presence of active regions $S$ and a reference value $S_{0}$ is given by:

$$
\begin{aligned}
S-S_{0}= & S_{0} C \sum_{i: \mu_{i} \geq 0} A_{i} \mu_{i}\left[a_{\mathrm{p}}+b_{\mathrm{p}} \mu_{i}+c_{\mathrm{p}} \mu_{i}^{2}\right] \\
& \times\left[\left(c_{\mathrm{s}}-1\right)+Q\left(c_{\mathrm{f}}+c_{\mathrm{f}}^{\prime} \mu_{i}-1\right)\right]+S_{\mathrm{r}}
\end{aligned}
$$

where $A_{i}$ is the area of the $i$ th active region in unit of the solar surface; $\mu_{i} \equiv \cos \psi_{i}$, where $\psi_{i}$ is the limb (or foreshortening) angle, i.e., the angle between the normal to the $i$ th active region and the line of sight; $a_{\mathrm{p}}, b_{\mathrm{p}}$ and $c_{\mathrm{p}}$ are the coefficients that give the bolometric specific intensity of the photosphere as a function of the limb angle adopting a quadratic limb-darkening law; $C \equiv\left[(1 / 4)\left(a_{\mathrm{p}}+2 b_{\mathrm{p}} / 3+c_{\mathrm{p}} / 2\right)\right]^{-1}$; $c_{\mathrm{S}}$ is a coefficient specifying the sunspot bolometric contrast; $c_{\mathrm{f}}$ and $c_{\mathrm{f}}^{\prime}$ are the coefficients specifying the facular bolometric contrast; $Q$ is the ratio between the area of the faculae and the area of the sunspots in an active region; and $S_{\mathrm{r}}$ is an additive term that takes into account the time variability of the contribution of the uniformly distributed network to the solar irradiance. The contribution of the active regions on the hemisphere opposite to the observer is not included in the summation because it is extended to the regions having $\mu_{i} \geq 0$. Active regions are considered to be point-like brightness inhomogeneities for the treatment of their rising and setting at the limb, which is justified in the solar case because their areas do not exceed $1-2 \%$ of the surface. The coefficients of the quadratic limb darkening law adopted to describe the unperturbed bolometric specific intensity are: $a_{\mathrm{p}}=0.36, b_{\mathrm{p}}=0.84$ and $c_{\mathrm{p}}=-0.20$ (Pierce 2000), which yields: $C=4.88$. The bolometric contrast of the sunspots was adopted to be equal to the value of model II(A) of Sofia et al. (1982) and independent of the position on the solar disk: $c_{\mathrm{s}}=0.67$. Our model does not distinguish between umbra and penumbra and adopts a mean sunspot structure at an intermediate temperature as in previous modelling approachs (cf. Chapman 1987; Unruh et al. 1999). A computation based on Kurucz's atmospheric models (Kurucz 2000) gives a bolometric contrast of 0.64 , which changes of only a few percents from the disk center to the limb, by adopting an unperturbed photosphere with an effective temperature $T_{\text {eff }}=5780 \mathrm{~K}$ and $\log g=4.44\left(\mathrm{~cm} \mathrm{~s}^{-2}\right)$, a sunspot photosphere with $T_{\text {eff }}=5150 \mathrm{~K}$, and the same gravity (cf. Unruh et al. 1999). The bolometric facular contrast coefficients are: $c_{\mathrm{f}}=1.115$ and $c_{\mathrm{f}}^{\prime}=-0.115$ according to Foukal et al. (1991). In our model each active region consists of an area $A_{i}$ covered with sunspots and an area $Q A_{i}$ covered with faculae. The photometric effect of the spots is maximum at the disk center where their projected area is at its maximum, whereas the photometric contribution of the faculae is a more complicated function of the position being the facular contrast maximum at the limb. The ratio of the facular to sunspot area was assumed to be $Q=10$ and held fixed in our model.

Solar observations show that the contrast of faculae and, to a lesser extent, of the sunspots varies significantly among active regions (Chapman 1987; Chapman et al. 1994). Moreover, systematic changes with the phase of the solar cycle are also possible (Albregtsen et al. 1984). In particular, the average value of $Q$ is a function of the phase of the solar cycle, with $Q$ decreasing for larger active regions appearing around the maximum of activity (Foukal 1998). We did not include these effects in our model because we are interested in analysing the TSI by adopting a simple stellar-like approach. In the case of the other stars, we have no a priori information on the phase of the activity cycle or the faculae-to-cool-spots area ratio.

The value of the reference bolometric irradiance $S_{0}$, which corresponds to the solar flux without active region and uniform network contributions, is also unknown in the stellar case. $S_{0}$ is the value with respect to which the model TSI is normalized before fitting the observed variations. In modelling stellar wideband light curves it is customary to adopt as a reference value the flux corresponding to the brightest magnitude of the star in the considered time series, i.e., at the minimal coverage of the stellar disk by cool spots (cf., e.g., Messina et al. 1999). In the present analysis, we adopt a stellar-like approach and assume $S_{0}=1367.3 \mathrm{~W} \mathrm{~m}^{-2}$, i.e., the maximum TSI value in our time series. It is important to note that the values of the active region areas $A_{i}$ 's and of the uniformly distributed network contribution $S_{\mathrm{r}}$ are referred to such a value of the solar TSI. However, their variations are largely independent of $S_{0}$ and represent the truly meaningful quantities to characterize the stellar activity behaviour, as will be shown by our modelling results (cf. Sect. 4).

The position of an active region on the Sun is defined by its longitude $\lambda_{i}$ and latitude $\theta_{i}$ in the Carrington reference frame. The value of $\mu_{i}$ is given by:

$\mu_{i}=\cos i_{\odot} \sin \theta_{i}+\sin i_{\odot} \cos \theta_{i} \cos \left(\lambda_{i}+\Omega t-L_{0}\right)$

where $i_{\odot}$ is the inclination of the rotation axis of the Sun with respect to the line of sight, $\Omega$ the angular velocity of the Sun, $t$ the time and $L_{0}$ the longitude of the central meridian of the solar disk. It is important to note that the inclination of the solar rotation axis changes along the year due to the orbital motion of SoHO around the Sun. More precisely, if $B_{0}$ is the latitude of the center of the disk of the Sun: $i_{\odot}=90^{\circ}-B_{0}$. In consideration of the small dimensions of the halo orbit of SoHO around the L1 point of the Sun-Earth system with respect to its orbit around the Sun, we can assume for $B_{0}$ and $L_{0}$ the values computed for a geocentric observer (Domingo et al. 1995).

It is important to note that the angular velocity of rotation of the reference frame of our model $\Omega$ does not coincide with that of the Carrington reference frame. Actually, it is an additional free parameter of the model because the rotational modulation of the solar flux by the active regions does not show a constant periodicity during the time interval we analysed, as shown in Sect. 2.

An important point regards the choice of the number of active regions to be adopted to model the TSI rotational 
modulation. A simple semi-sinusoidal modulation can be modelled by only one active region, but the observed modulation is usually of considerably more complex shape. This implies that at least two or, more frequently, three active regions are needed to obtain a good fit of the TSI modulation.

The analysis of the most accurate stellar light curves obtained so far supports such a choice also in the case of magnetically active rotators (Budding 1995). Moreover, the validity of the assumption of three active regions is confirmed a posteriori by the goodness of fit to the entire TSI time series (cf. Sect. 4). Therefore, our model requires the specification of eleven free parameters, that is the area and the coordinates of each active region plus the background irradiance $S_{\mathrm{r}}$ and the period of rotation of the pattern of the active regions $P \equiv 2 \pi / \Omega$. Their best values are evaluated by fitting the model to the observed TSI, $S_{\text {obs }}$, by minimizing the $\chi^{2}$ statistics:

$\chi^{2}=\frac{1}{M} \sum_{j=1}^{M} \frac{\left[S_{\mathrm{obs}}\left(t_{j}\right)-S\left(t_{j}\right)\right]^{2}}{\sigma^{2}}$,

where $M$ is the number of observations in the considered interval of time, $S_{\mathrm{obs}}\left(t_{j}\right)$ the observed value of the TSI at time $t_{j}$, $S\left(t_{j}\right)$ the value of the TSI evaluated from Eq. (1) at the same time, and $\sigma$ the standard deviation of the TSI measurements. We assume $\sigma / S_{0}=2.0 \times 10^{-5}$, to be constant for all the data set.

In order to fit the observations with our model, it is important to consider a data subset spanning a time interval shorter than the time scale for a significant evolution of the active regions on the Sun. After a series of tests, we found that a time interval of 14.0 days is the best compromise between the requirement of avoiding significant active region changes and that of sampling adequately the rotational modulation of the active regions themselves. As a matter of fact, the evolution of the active regions is so fast on the Sun that it is usually impossible to model adequately an entire rotation by using a fixed active region pattern, whereas with our choice of the length of the subset interval the fit turned out to be always good.

It is important to note that a numerical instability may arise in the fitting of a given data set because a linear combination of active region longitude and angular velocity appears in Eq. (2). Therefore, we have always fitted each data set by fixing a test value for the rotation period and then finding the best values of the remaining parameters that minimized the $\chi^{2}$ by applying the constrained minimization algorithm by Byrd et al. (1995), as implemented by Zhu et al. (1994). Then the rotation period $P$ was iteratively adjusted in the interval $23.0-33.5 \mathrm{~d}$ by performing successive fitting to locate the absolute minimum of the $\chi^{2}$ by means of a golden section algorithm (Press et al. 1992).

We used the best fit parameters of a given data set as the starting point for the analysis of the successive set, by adopting a spacing between consecutive sets of $7.0 \mathrm{~d}$, unless a larger gap was imposed by the lack of data. In this way we could follow the evolution of the pattern of active regions on a time interval shorter than that adopted to sample their rotational modulation. This procedure led to a further improvement of the quality of the fit.
It is worth noticing that the parameters we held fixed specify the limb-darkening of the unperturbed stellar disk and the contrast of the surface inhomogeneities. They are illconstrained by bolometric flux variations alone. In fact, only multi-band data may provide information for their determination (cf. Sect. 5). On the other hand, the geometrical parameters specifying the active region areas and coordinates and the uniform network contributions are better-constrained by the TSI modulation and are assumed as variable parameters in our fitting procedure.

From a mathematical point of view, the solution obtained by minimizing the $\chi^{2}$ is usually unique when the TSI time variation is dominated by the rotational modulation of a few and stable active regions, and the contrast and limb-darkening parameters are known a priori as well as the inclination of the rotation axis and the rotation period (e.g., Eker 1996). Conversely, when the TSI variation is produced by a complex pattern of many small active regions or is greatly affected by the fluctuations due to active region growth and decay, multiple solutions may be found and the convergence and stability of the fitting algorithm may not be warranted.

Our procedure of fitting the geometrical parameters of the active regions only for fixed values of $P$ and then minimizing the $\chi^{2}$ with respect to $P$, eliminates a primary source of instability in the convergence procedure. However, in order to obtain a locally stable solution, we found that also the values of the active region areas $A_{i}$ 's and the contribution of the uniformly distributed network $S_{\mathrm{r}}$ must be constrained from the outset. Otherwise, there can be a trade-off between the active region total area and the variation of $S_{\mathrm{r}}$, which makes the resulting solution non-unique and unstable. This is a well known inconvenient in the analysis of wide-band light curve sequences and we adopted the same method used in stellar light curve analysis to overcome the problem. Specifically, the depth of the largest dip of the TSI time series was used to fix limits for the spot area: $0 \leq A_{i} \leq 8.2 \times 10^{-4}$; while the range spanned by the upper envelope of the TSI series in the 1996-2001 interval was used to constrain the variation of $S_{\mathrm{r}}:-1.5 \times 10^{-4} \leq S_{\mathrm{r}} / S_{0} \leq 1.5 \times 10^{-4}$.

The above mentioned constraints proved to be sufficient to warrant convergence and local stability for the determination of the geometrical free parameters in the case of three active regions. However, from a global point of view, sometimes multiple solutions with comparable values of the $\chi^{2}$ may be present and the fitting algorithm may become unstable when their minima are so close that their basins of attraction intersect one with the other. Actually, we found that our best fitting procedure converged in more than $80 \%$ of the cases by adopting as an initial guess, for the analysis of a given TSI subset, the best fit parameters of the previous subset. In the remaining cases, we needed to change the initial values of the parameters to avoid the solution to get stuck around a local minimum with an unacceptably high value of the $\chi^{2}$. In these cases, by applying repeatedly a bootstrap routine for the choice of new initial parameters (i.e., starting values of the active region areas and longitudes), we searched for other minima in the $\chi^{2}$ landscape until a solution with an acceptable $\chi^{2}$ value was found. Such a multi-stage procedure yielded a good fit in all the cases, i.e., for 

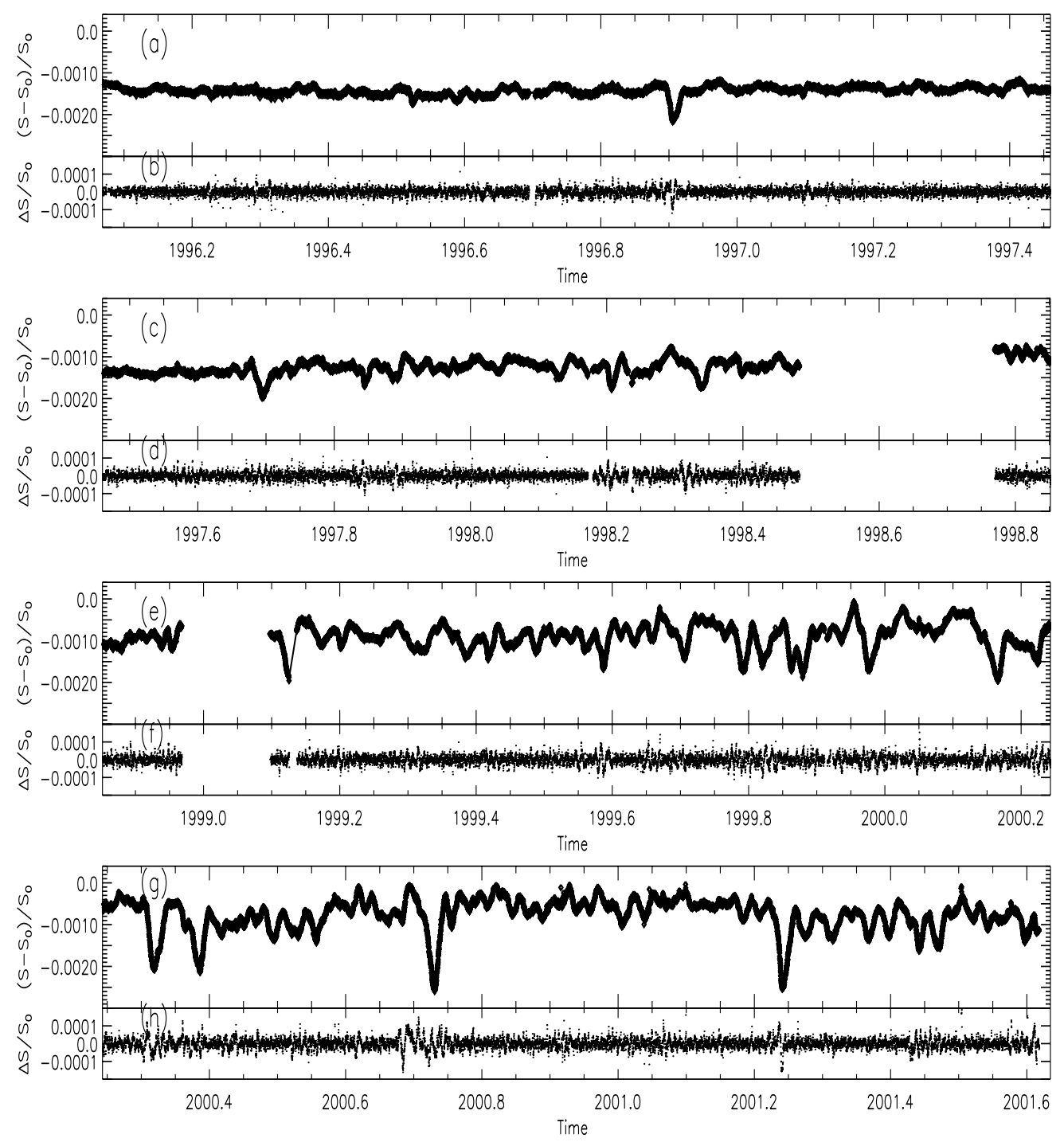

Fig. 3. a) The TSI data sequence for the time interval from mid-January 1996 to June 1997 (open diamond) and the best fit computed with our model (solid line); b) the deviation of the best fit from the TSI data in the same time interval; c) the TSI data sequence from June 1997 to October 1998 (open diamond) and the best fit (solid line); d) the deviation of the best fit from the TSI data in the same time interval; e) the TSI data sequence from October 1998 to March 2000 (open diamond) and the best fit (solid line); f) the deviation of the best fit from the TSI data in the same time interval; g) the TSI data sequence from March 2000 to August 2001 (open diamond) and the best fit (solid line); h) the deviation of the best fit from the TSI data in the same time interval. The model is always embedded into the data sequence except when gaps are present (cf., e.g., the interval 1999.12-1999.13).

all the analysed TSI subsets ranging from mid January 1996 to the end of August 2001.

\section{Results}

The observed relative variation of the TSI together with our model best fit is plotted in Fig. 3 with a zoom-in to selected time intervals shown in Fig. 4. The value of the fit for a given datum was computed with the parameters determined by the best fit of the 14-d data subset the midpoint of which was the closest preceding the datum. Thus the time interval between a given datum and the nominal epoch of the best fit parameters was always smaller than $7.0 \mathrm{~d}$. Of course, successive fits had slightly different endpoints, but their relative differences did not exceed $5 \times 10^{-5}$ and were randomly distributed. We chose to retain such small differences and the small changes in the slopes of the fitting curves near the endpoints to avoid the introduction of any forced matching in the data space, which should have been difficult to justify on a geometrical or physical ground. In any case, the cadence of the endpoint mismatchs can be easily changed by modifying the time interval between successively fitted data sets. The fit computed according to these prescriptions was always good with relative deviations from the data $<10^{-4}$, during the activity minimum phases, and of $\sim 2 \times 10^{-4}$ during the phases with the largest active regions, close to cycle 23 maximum. Such deviations were due to the growth and decay of small active regions on time scales of the order of a day that could not be modelled by our approach because the active region configuration we adopted was changed every seven days. 

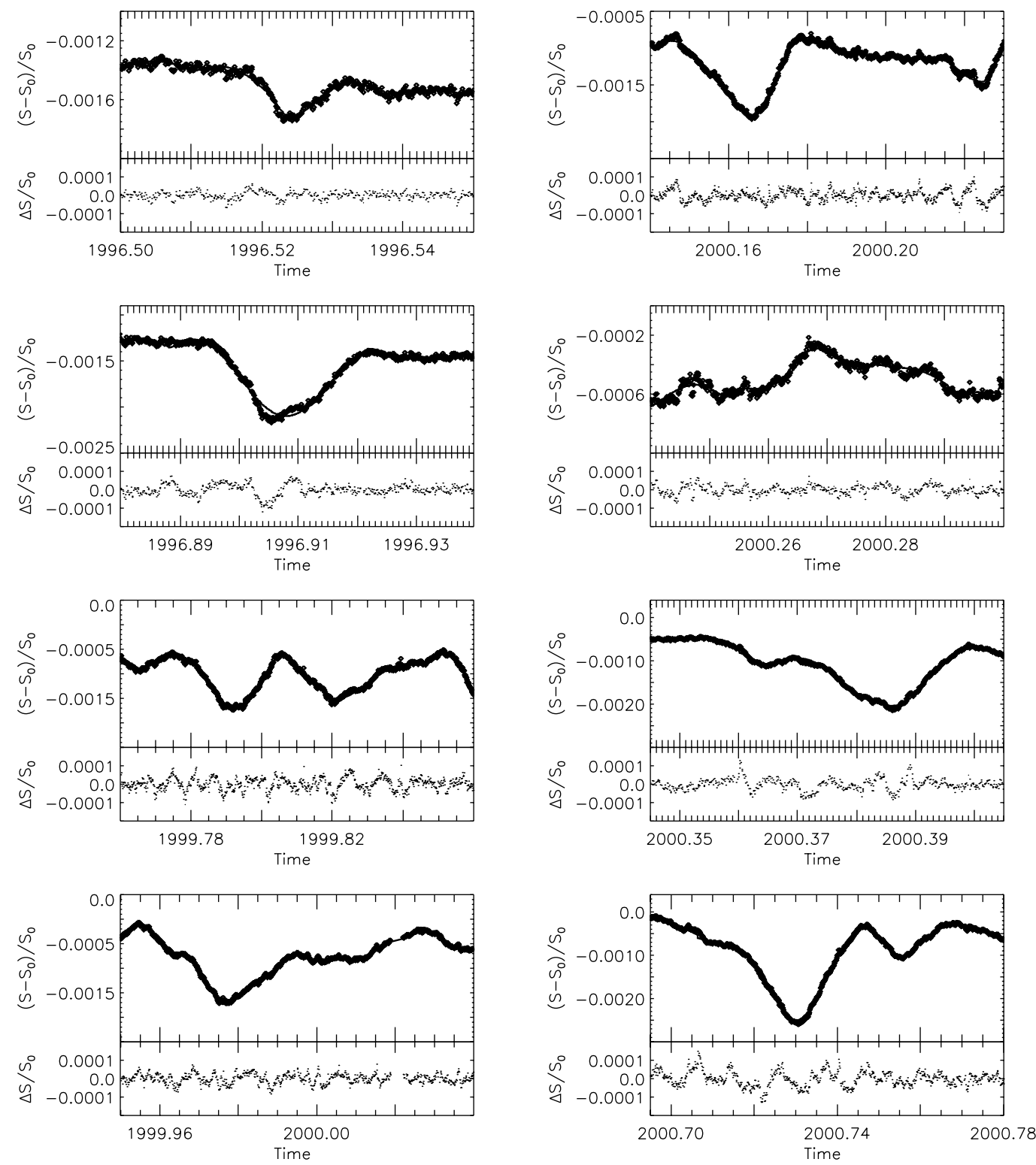

Fig. 4. A zoom-in to eight short time intervals along the whole data sequence plotted in Fig. 3. In each couple of panels, the upper one shows the TSI data (open diamonds) and the best fit computed with our model (solid line) vs. time, whereas the lower one shows the deviation between data and fit vs. time. The sequence of panel couple runs from activity cycle minimum (upper left couple) to the rising phase of cycle 23 (lower left couple) and then from the rising phase (upper right couple) to activity maximum of cycle 23 (lower right couple). The model is always embedded into the data sequence, except when data gaps or a significant deviation is present (cf., e.g., the 1996.89-1996.93 time interval in the second couple of panels on the left column).

The $\chi^{2}$ of the best fits to the individual data subsets were plotted in Fig. 5. Most of the values were around the unity. However, significantly larger values of the $\chi^{2}$ were obtained for the data sets with the largest active complexes around the activity maximum. This is likely to be due to the evolution of several short-lived active regions that could not be modelled by our approach.

The best values of the rotation period, as derived by means of our fitting procedure, are plotted in Fig. 6. They show a large dispersion around a mean value of $30.25 \mathrm{~d}$ and no systematic trend with the solar cycle is apparent. A time baseline of fourteen days is certainly not adequate to measure the rotation period with high accuracy. Moreover, the large variations between the fits to successive data subsets suggest that our code tried to deal with the growth and decay of the active regions by changing the period of rotation of the model star in order to vary their projected area during the transit over the disk and their epochs of rising and setting at the limb. The fact that the average period is about $10 \%$ longer than the synodic period of rotation of the Sun is due to the accumulation of the period values toward the upper limit for $P_{\text {rot }}$ that was fixed at $33.5 \mathrm{~d}$. This indicates that, on the average, the photometric effect of a model active region tends to persist for a somewhat longer time than the actual rotation period of the Sun, an effect that may be related to our assumption that spots and faculae were co-spatial and that the extension of the active regions was negligible. 